\title{
Is Heart Rate Recovery Really Associated with Severity of Coronary Artery Disease in Patients with Normal Exercise Treadmill Test?
}

\section{Reza Na isi Moghadam¹, Masoud Kamalian Mehrizi², Aryan Naghedi ${ }^{3}$, Nasim Namiranian ${ }^{4}$, Amir Pasha Amel Shahbaz $^{5}$, and Seid Kazem Razavi-Ratki ${ }^{6, *}$}

${ }^{1}$ Associate Professor of Radiology, Department of Radiology, Faculty of Medicine, Shahid Sadoughi University of Medical Sciences, Yazd, Iran 2Department of Radiology, Shahid Sadoughi Hospital, Faculty of Medicine, Shahid Sadoughi University of Medical Sciences, Yazd, Iran

${ }^{3}$ Department of Cardiology, Shahid Sadoughi Hospital, Shahid Sadoughi University of Medical Sciences, Yazd, Iran

${ }^{4}$ Assistant Professor of Community and Preventive Medicine, Yazd Diabetes Research Center, Shahid Sadoughi University of Medical Sciences, Yazd, Iran

${ }^{5}$ Assistant Professor of Radiology, Department of Radiology, Faculty of Medicine, Shahid Sadoughi University of Medical Sciences, Yazd, Iran

${ }^{6}$ Assistant Professor of Nuclear Medicine, Department of Radiology, Faculty of Medicine, Shahid Sadoughi University of Medical Sciences, Yazd, Iran

*Corresponding author: Razavi-Ratki SK, Assistant Professor of Nuclear Medicine, Department of Radiology, Faculty of Medicine, Shahid Sadoughi University of Medical Sciences, Yazd, Iran, E-mail: sk.razavi@ssu.ac.ir

Received: 14 Feb, 2020 | Accepted: 03 Mar, 2020 | Published: 09 Mar, 2020

Citation: Moghadam RN, Mehrizi MK, Naghedi A, Namiranian N, Shahbaz APA, et al. (2020) Is Heart Rate Recovery Really Associated with Severity of Coronary Artery Disease in Patients with Normal Exercise Treadmill Test? J Hear Health 6(1): dx.doi.org/10.16966/2379-769X.153

Copyright: ( 2020 Moghadam RN, et al. This is an open-access article distributed under the terms of the Creative Commons Attribution License, which permits unrestricted use, distribution, and reproduction in any medium, provided the original author and source are credited.

\begin{abstract}
Background and Objectives: Rapid diagnosis and treatment of cardiovascular diseases is really helpful in reducing further complications. There are different modalities for this goal but each has benefits and limitations. Exercise Treadmill Test (ETT) is a non-invasive, cheap and accessible test. Coronary CT Angiography (CTA) is recently considered as an applied modality for assessing severity of Coronary Artery Disease (CAD). In this study we aimed to investigate possible association between Heart Rate Recovery (HRR) in ETT and severity of CAD in CTA.

Materials and Methods: In this cross-sectional, descriptive-analytic study we enrolled 70 susceptible patients for coronary artery disease. All patients underwent ETT and CTA. HRR in $1^{\text {st }}$ and $2^{\text {nd }}$ minute along with severity of CAD detected in CTA and patient's demographic information were recorded in a questionnaire. Finally, all data was analyzed using SPSS version 21.0 software.

Results: In this study, $43(61.4 \%)$ of patients were men. Mean HRR in $1^{\text {st }}$ and $2^{\text {nd }}$ minutes was $20.9 \pm 7.36$ and $35 \pm 10.10$ respectively. 27 of patients (38.5\%) had abnormal HRR. We observed a statistically significant association between $1^{\text {st }}$ and $2^{\text {nd }}$ minute HRR and Hypertension (HTN). We also observed a significant association between severity of CAD with HTN, Diabetes Mellitus (DM) and Hyperlipidemia (HLP). A significant association was observed between severity of $C A D$ and $1^{\text {st }}$ minute $(P$-value $=0.01)$ and $2^{\text {nd }}$ minute $(P$-value $=0.002)$ HRR in this investigation.

Conclusion: Considering the association between HRR in ETT and severity of CAD in CTA along with benefits of ETT such as non-invasive entity, lower expenses and availability, it seems logical to suggest ETT for patients before CTA.
\end{abstract}

Keywords: Autonomic nervous system; Coronary artery disease; Coronary CT angiography; Exercise treadmill test; Heart rate recovery

\section{Introduction}

Autonomic Nervous System (ANS) adjusts electrical and mechanical activity of heart myocardium by maintaining balance between sympathetic and parasympathetic systems [1]. Heart rate fluctuates based on sympathetic and parasympathetic activity. Short term and long term heart rate changes reflect ANS function. Impaired ANS function leads to imbalance in adjusting cardiovascular function and causes autonomic cardiac neuropathy [2].
Heart Rate Variability (HRV) is an important physiologic factor in human body which its activity enhances physical wellbeing and also reduces tension and anxiety. HRV is a quantitative variable for evaluating ANS function. Recently HRV is reported to be a suitable predictor for cardiovascular involvement and autonomic neuropathy in scientific literature [3].

Exercise Treadmill Test (ETT) is a cheap, non-invasive, low risk and highly available test for diagnosis of Coronary Artery Diseases 
(CAD) and provides very useful information for physicians. Among applied findings of ETT, Heart Rate Recovery (HRR) and Exercise Capacity (EC) are mentionable.

HRR is number of reduced heart beats after finishing ETT which is often measured after one minute and is considered as a good predictor for cardiovascular involvement and autonomic neuropathy. HRR more than 18 beats per minute is generally considered as normal and is favorable. Predictive value of HRR is independent of existence of coronary artery disease, consumption of $\beta$-blocker agents and positive or negative results of ETT.

EC is also defined as maximum activity that each individual can reach during ETT. In order to unificate this index, Metabolic Equivalents (METs) are used. Each MET unit is equal to amount of activity in which $3.5 \mathrm{~mL}$ of oxygen is burnt per each $\mathrm{Kg}$ of body weight per minute. ETT devices measure patient's activity and export it in MET based on selected protocol, speed and incline. Recent studies conclude that EC and HRR are powerful and independent predictors for risk of mortality due to cardiovascular diseases [4].

ETT is known as a non-invasive routine test for screening patients susceptible for coronary artery diseases but this test is not accurate enough and several false negative or false positives are reported in ETT [5].

Nowadays Coronary CT Angiography (CTA) is applied as an up to date non-invasive modality for diagnosis of cardiovascular diseases. In this imaging modality artifacts caused by motion and calcified plaques sometimes makes it hard for clinicians to make a decision [6].

There are few studies carried out to find any possible association between CTA results and HRR after ETT. Based on mentioned above, in this study we aimed to investigate existence of any association between CTA results and HRR after ETT in patients with moderate to severe risk of coronary artery stenosis.

\section{Materials and Methods}

This study is a cross-sectional, descriptive analytic study in which we enrolled 70 consecutive patients with moderate to severe risk for coronary artery involvement referred to Afshar heart center, Yazd, Iran for CTA between December 2018 and December 2019. An informed consent was obtained from all patients and the study was designed and performed based on latest declaration of Helsinki. This research is registered in research ethics committee of Shahid Sadoughi University of medical sciences, Yazd, Iran with IR.SSU.MEDICINE.REC.1398.252 approval ID.

With a power of $80 \%$ and confidence interval of $95 \%$ and $\alpha=5 \%$ a number of 70 patients was calculated to be suitable for this study.

All patients referred for CTA who previously underwent ETT if carrying normal results, were included in this study. Those patients with contraindication for ETT, history of Percutaneous Coronary Intervention (PCI) or Coronary Artery Bypass Grafting (CABG), renal failure, history of consumption of medical agents interfering heart rate such as $\beta$-blockers and those who cannot finish ETT were excluded.

All patients underwent ETT with standard protocol. ETT continued 1 minute after patients achieved $85 \%$ of maximum heart rate and HRR was measured 1 minute and 2 minutes after finishing ETT. Minimum and maximum heart rate plus mean HRV was registered and results were compared with CTA findings.

All CTA were performed using a 320 slice multidetector device and $0.4 \mathrm{mg}$ nitroglycerine was administered for all patients 2 minutes before imaging and all images were acquired while breath holding.
Patient's demographic information and history and HRR after ETT and CTA findings were all entered a questionnaire and finally data was analyzed using SPSS version 21.0 software.

\section{Statistical analysis}

For reporting descriptive statistics, we used frequency tables and descriptive tests as mean $\pm \mathrm{SD}$, minimum, maximum and percentage. A stacked bar chart is used for better demonstrating the association between severity of CAD and HRR.

To evaluate the association between $1^{\text {st }}$ and $2^{\text {nd }}$ minute HRR and dichotomous variables such as gender, diabetes mellitus, hypertension, hyperlipidemia and smoking, independent t-test was used and for severity of CAD as a trichotomous variable, one-way ANOVA with Bonferroni post hoc test was applied. Results of these tests are reported as P-value plus $95 \%$ confidence interval.

Chi-square test was used to assess the relationship between severity of coronary artery disease and other nominal variables including diabetes mellitus, hypertension, hyperlipidemia and smoking and the results are reported as P-value.

In all statistical tests a $95 \%$ confidence interval is reported and a P-value less than 0.05 are considered to be statistically significant.

\section{Results}

Our study population consisted of 43 (61.4\%) men and 27 (38.6\%) women. $18(25.7 \%)$ of patients had diabetes mellitus, $35(50 \%)$ had hypertension, $42(60 \%)$ had dyslipidemia and 23 (32.9\%) were smokers. In this study 33 (47.1\%), 22 (31.45\%), $12(17.1 \%)$ and 3 (4.3\%) of patients had $0,1,2$ and 3 vessel involvement.

In this study latest in test heart rate was recorded to be $139.4 \pm$ 16.74. First minute HRR was $20.9 \pm 7.36$ and second minute HRR was $35 \pm 10.10$. Table 1 shows descriptive statistics related to HRR in our study population.

Categorizing patients based on coronary artery involvement shows that $32(45.7 \%)$ patients were normal, $24(34.3 \%)$ of them had mild involvement and $14(20 \%)$ patients had moderate to severe coronary artery involvement.

Table 2 indicates mentioned above statistics as a table.

In this study, among a total of 70 patients, 27 (38.5\%) patients had abnormal HRR. Our results show that $53.49 \%$ of patients with normal CTA have a normal HRR; this is while only $6.97 \%$ of patients with moderate to severe coronary artery involvement have a normal HRR.

Table 1: Minimum, maximum and mean heart rate variability in study population.

\begin{tabular}{|l|c|c|c|}
\hline & Minimum & Maximum & Mean \pm SD \\
\hline Latest in-test HR & 95 & 171 & $139.4 \pm 16.74$ \\
\hline $\mathbf{1}^{\text {st }}$ minute HRR & 5 & 40 & $20.9 \pm 7.36$ \\
\hline $\mathbf{2}^{\text {nd }}$ minute HRR & 12 & 52 & $35 \pm 10.10$ \\
\hline
\end{tabular}

Table 2: Descriptive statistics related to severity of coronary artery involvement.

\begin{tabular}{|l|c|c|}
\hline \multicolumn{1}{|c|}{$\begin{array}{c}\text { Severity of coronary artery } \\
\text { involvement }\end{array}$} & Frequency & Percentage \\
\hline Normal & 32 & $45.7 \%$ \\
\hline Mild & 24 & $34.3 \%$ \\
\hline Moderate to severe & 14 & $20 \%$ \\
\hline
\end{tabular}


Figure 1 below shows distribution of normal and abnormal HRR among different patient's groups based on severity of CAD.

In this study we evaluated the association between HRR in $1^{\text {st }}$ and $2^{\text {nd }}$ minute with gender, Diabetes Mellitus (DM), Hypertension (HTN), Dyslipidemia (HLP), smoking and of course severity of coronary artery disease. The results are summed up in table 3 below. Our results indicate that there is a statistically significant association between severity of coronary artery disease and $1^{\text {st }}$ minute HRR $(\mathrm{P}$-value $=0.01)$ and $2^{\text {nd }}$ minute HRR (P-value $\left.=0.002\right)$.

We also assessed the association between severity of coronary artery disease and DM, HTN, HLP and smoking using Chi-square test. Table 4 shows associated $\mathrm{P}$-values of each Chi-square test performed to assess the relationship between severity of CAD and other risk factors.

\section{Discussion}

In this study we found that latest in-test heart rate was $139.4 \pm$ 16.7 and mean $1^{\text {st }}$ minute and $2^{\text {nd }}$ minute HRR were $20.9 \pm 7.36$ and $35 \pm 10.10$ respectively. Our results show that 27 (38.5\%) of patients had abnormal HRR. In a study performed by Ghaffari S, et al. on 140

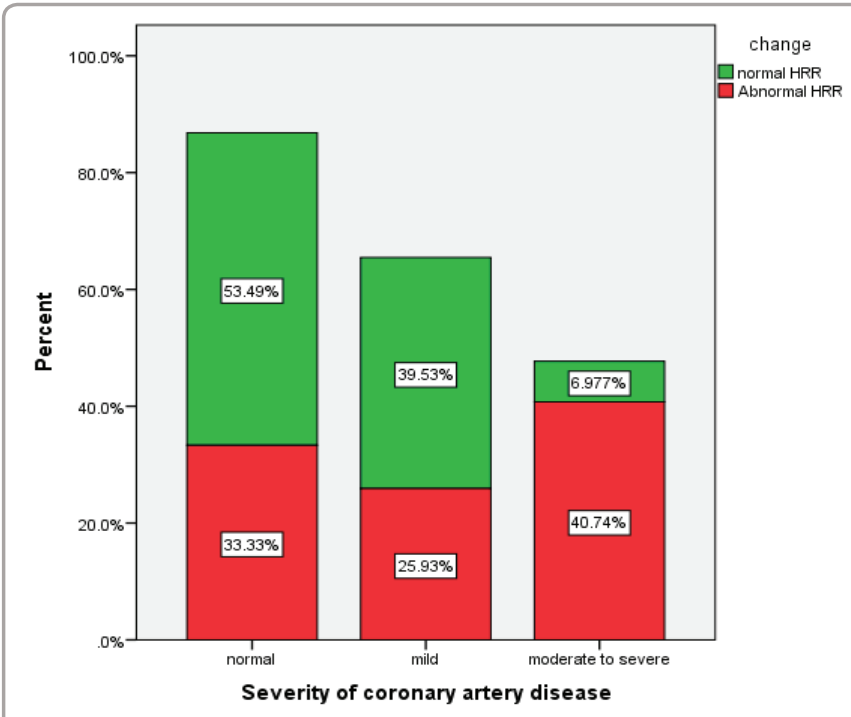

Figure 1: Distribution of normal and abnormal HRR among different study groups based on severity of CAD.

Table 3: Association between $1^{\text {st }}$ and $2^{\text {nd }}$ minute HRR and studied variables.

\begin{tabular}{|l|c|c|c|c|}
\hline \multirow{2}{*}{\multicolumn{1}{c|}{ Variable }} & \multicolumn{2}{|c|}{$\mathbf{1}^{\text {st }}$ minute HRR } & \multicolumn{2}{c|}{$\mathbf{2}^{\text {nd }}$ minute HRR } \\
\cline { 2 - 5 } & P-value & $\mathbf{9 5 \%} \mathbf{C l}$ & P-value & $\mathbf{9 5 \%} \mathbf{~ C l}$ \\
\hline Gender & 0.21 & $-5.85,1.33$ & 0.08 & $-8.72,0.54$ \\
\hline DM & 0.06 & $-0.28,7.61$ & 0.29 & $-3.11,9.96$ \\
\hline HTN & $\mathbf{0 . 0 0 5}$ & $1.51,8.19$ & $\mathbf{0 . 0 0 0 1}$ & $4.27,13.03$ \\
\hline HLP & 0.93 & $-3.75,3.47$ & 0.61 & $-3.70,6.18$ \\
\hline Smoking & 0.30 & $-1.81,5.66$ & 0.45 & $-3.20,7.08$ \\
\hline Coronary artery disease & $\mathbf{0 . 0 1}$ & $-11.72,-0.63$ & $\mathbf{0 . 0 0 2}$ & $-18.20,-3.45$ \\
\hline
\end{tabular}

Table 4: Association between severity of Coronary Artery Disease (CAD) and other variables.

\begin{tabular}{|l|c|c|c|c|}
\hline & DM & HTN & HLP & Smoking \\
\hline $\begin{array}{l}\text { Severity } \\
\text { of CAD }\end{array}$ & P-value=0.015 & P-value=0.001 & P-value=0.01 & P-value=0.14 \\
\hline
\end{tabular}

patients they also reported $13.7 \%$ abnormal HRR in their population which is similar to our results [7]. Different studies have reported that HRR is a predictive index for cardiovascular death and indicates autonomic dysfunction [8-11].

In our population, CTA results showed that $4.57 \%$ of patients did not have any coronary artery involvement which is similar to $41 \%$ which was reported by Lau G, et al. in 2013 [12].

By checking literature, we found a lot of information related to association between ETT and severity of CAD with studied risk factors. Although there are little differences in results, but it seems that most of them somehow confirm our results. The associations between severities of CAD with HTN [13-17], gender [11,18,19], DM [20-24], HLP [20-22] and smoking [20,25] are repeatedly reported in published articles.

Our clinical challenge was not that much repeated and is novel in its kind. In clinics, HRV is not taken that serious as other mentioned risk factors above. By this study we wanted to emphasize on importance of this index in predicting severity of coronary artery disease. We try to bold this marker at the same level as other risk factors such as HTN, DM, etc.

In a study performed by Chinnaiyan KM, et al., in 2012, researchers reported that there is a statistically significant correlation between ETT results and CTA findings [25].

Ghaffari S, et al. also carried out a similar study on 140 patients undergoing ETT. They concluded that there is a significant relationship between abnormal $1^{\text {st }}$ minute HRR and severity of coronary artery involvement [7]. Having these findings in mind it can be concluded that ETT is a non-invasive, cheap, simple and accessible test and has fewer complications compared to other modalities and it can be applied as a first step diagnostic test.

In this study we did not focus on prognosis of patients but Cho I, et al. reported that ETT is more useful than CTA in evaluating prognosis of patients with moderate to severe coronary artery disease. Based on researcher's opinion along with radiation risk, higher expenses and other disadvantages of CTA, selecting this modality as a first line test should be postponed [26].

Blankstein R, et al. compared ETT and CTA in patients with acute chest pain in 220 consecutive patients. The concluded that positive ETT possess a sensitivity of $30 \%$ and specificity of $93 \%$ and can be very useful for diagnosis of more than $50 \%$ coronary artery obstruction specially in younger patients [27].

In opposition with our study and similar studies mentioned above Ahn JH, et al. [28] and Nørgaard KS, et al. [29] reported that CTA has a higher sensitivity and specificity compared to ETT. They suggested that this technique can be used as a first line diagnostic test in patients susceptible for coronary artery disease.

Considering this disagreement on literature, it seems that further researchers with higher study population and more accurate methods and better designs can be really problem solving in clinical decision making in approaching patients with susceptible coronary artery disease.

Same as any other scientific research, our research has also its own limitations. In this study we could only include 70 patients which is not a large study population but it was inevitable because of number of patients referred to only one center. Carrying out similar studies in a multi-disciplinary manner may solve this issue. In this study, patients were divided into 3 groups based on CTA findings according to 
cardiologist and radiologist opinions. Although they were professional and experienced but applying a unit and quantitative indicator such as coronary calcium score is strongly recommended to future researchers. By having a larger study population and more quantitative data, performing a more accurate and comprehensive statistical analysis such as regression models will be possible which can be a clue for our colleagues.

\section{Conclusion}

Based on our findings, there is a statistically significant association between $1^{\text {st }}$ minute and $2^{\text {nd }}$ minute HRR and CTA findings. There was also a relationship observed between HTN and HRR. We also found that DM, HTN and HLP are correlated with severity of coronary artery disease.

Considering non-invasive entity of ETT, lower price and higher availability of ETT, it seems really logical to suggest this modality in susceptible patients before undergoing coronary CTA.

\section{References}

1. Sztajzel J (2004) Heart rate variability: A noninvasive electrocardiographic method to measure the autonomic nervous system. Swiss Med Wkly 134: 514-522.

2. Matt D, Scheffel H, Leschka S, Flohr TG, Marincek B, et al. (2007) Dual-source CT coronary angiography: image quality, mean heart rate, and heart rate variability. AJR Am J Roentgenol 189: 567-573.

3. Zhang J, Fletcher JG, Scott Harmsen W, Araoz PA, Williamson EE, et al. (2008) Analysis of heart rate and heart rate variation during cardiac CT examinations. Acad Radiol 15: 40-48.

4. Gibbons RJ, Balady GJ, Bricker JT, Chaitman BR, Fletcher GF, et al. (2002) ACC/AHA 2002 guideline update for exercise testing: summary article: a report of the American College of Cardiology/ American Heart Association Task Force on Practice Guidelines (Committee to Update the 1997 Exercise Testing Guidelines). Circulation 106: 1883-1892.

5. Faisal AW, Abid AR, Azhar M (2007) Exercise Tolerance Test: A comparison between true positive and false positive test results. $J$ Ayub Med Coll Abbottabad 19: 71-74.

6. Vanhoenacker PK, Heijenbrok-Kal MH, Van Heste R, Decramer I, Van Hoe LR, et al. (2007) Diagnostic performance of multidetector $\mathrm{CT}$ angiography for assessment of coronary artery disease: metaanalysis. Radiology 244: 419-428.

7. Ghaffari S, Kazemi B, Aliakbarzadeh P (2011) Abnormal heart rate recovery after exercise predicts coronary artery disease severity. Cardiol J 18: 47-54.

8. Halon DA, Dobrecky-Mery I, Gaspar T, Azencot M, Yaniv N, et al. (2010) Heart rate recovery after exercise and coronary atheroma in asymptomatic individuals with type 2 diabetes mellitus: A study using 64-slice coronary CT angiography. Int J Cardiol 145: 102-103.

9. Ahad Shafie, Adel Goharimoghadam, Mahla sadat Nabavi Zadeh (2016) The effects of interval exercise training on heart rate recovery and heart rate variability after coronary artery bypass grafting. Razi Journal of Medical Sciences 23: 91-98.

10. Aubert AE, Seps B, Beckers F (2003) Heart rate variability in athletes. Sports Med 33: 889-919.

11. Pothineni NV, Shirazi LF, Mehta JL (2016) Gender differences in autonomic control of the cardiovascular system. Curr Pharm Des 22: 3829-3834.
12. Lau G, Rigolli M, Wickham J, Brannigan H, Christiansen J, et al. (2013) CT Coronary Angiography is a Useful Diagnostic Test Following Equivocal Exercise Stress ECG in Troponin-Negative Chest Pain. Heart Lung Circ 22: S171.

13. Zeina AR, Odeh M, Rosenschein U, Zaid G, Barmeir E (2008) Coronary artery disease among asymptomatic diabetic and nondiabetic patients undergoing coronary computed tomography angiography. Coronary artery Dis 19: 37-41.

14. Nakanishi R, Baskaran L, Gransar H, Budoff MJ, Achenbach S, et al. (2017) Relationship of hypertension to coronary atherosclerosis and cardiac events in patients with coronary computed tomographic angiography. Hypertension 70: 293-299.

15. Weber T, Lang I, Zweiker R, Horn S, Wenzel RR, et al. (2016) Hypertension and coronary artery disease: epidemiology, physiology, effects of treatment, and recommendations. Wien Klin Wochenschr 128: 467-479.

16. Wielemborek-Musial K, Szmigielska K, Leszczynska J, Jegier A (2016) Blood pressure response to submaximal exercise test in adults. Biomed Res Int 2016: 5607507.

17. Youn JC, Kang SM (2015) Cardiopulmonary exercise test in patients with hypertension: focused on hypertensive response to exercise. Pulse (Basel) 3: 114-117.

18. Mahon AD, Anderson CS, Hipp MJ, Hunt KA (2003) Heart rate recovery from submaximal exercise in boys and girls. Med Sci Sports Exerc 35: 2093-2097.

19. Fomin A, Ahlstrand M, Schill HG, Lund LH, Stahlberg M, et al. (2012) Sex differences in response to maximal exercise stress test in trained adolescents. BMC Pediatr 12: 127.

20. Bidel Z (2014) Evaluating the associations between some cardiovascular risk factors and coronary artery obstruction based on angiographic findings. J Ilam Univ Med Sci 22: 14-154.

21. Duda-Pyszny D, Trzeciak P, Gasior M (2018) Coronary artery disease in women. Kardiochir Torakochirurgia Pol 15: 44-48.

22. Karimzadeh Ghassab R, Hosseini Gohari L, Firoozrai M, Zavareii A, Basiri HA (2010) Levels of Small Dense LDL, Lipids and Lipoproteins in Patients with Coronary Artery Stenosis and Healthy Individuals. Razi Journal of Medical Sciences 16: 42-48.

23. Veeranna V, Pradhan J, Niraj A, Fakhry H, Afonso L (2010) Traditional cardiovascular risk factors and severity of angiographic coronary artery disease in the elderly. Prev Cardiol 13: 135-140.

24. Fox CS, Sullivan L, D'Agostino RB Sr, Wilson PW, Framingham Heart Study (2004) The significant effect of diabetes duration on coronary heart disease mortality: the Framingham Heart Study. Diabetes care 27: 704-708.

25. Chinnaiyan KM, Raff GL, Goraya T, Ananthasubramaniam K, Gallagher MJ, et al. (2012) Coronary computed tomography angiography after stress testing: results from a multicenter, statewide registry, ACIC (Advanced Cardiovascular Imaging Consortium). J Am Coll Cardiol 59: 688-695.

26. Cho I, Shim J, Chang HJ, Sung JM, Hong Y, et al. (2012) Prognostic value of multidetector coronary computed tomography angiography in relation to exercise electrocardiogram in patients with suspected coronary artery disease. J Am Coll Cardiol 60: 2205-2215.

27. Blankstein R, Ahmed W, Bamberg F, Rogers IS, Schlett CL, et al. (2012) Comparison of exercise treadmill testing with cardiac computed tomography angiography among patients presenting to the emergency room with chest pain: the Rule Out Myocardial Infarction Using Computer-Assisted Tomography (ROMICAT) study. Circ Cardiovasc Imaging 5: 233-242. 
28. Ahn JH, Park JR, Kim KH, Koh JS, Hwang SJ, et al. (2015) The prognostic value of exercise ECG test and cardiac computed tomography coronary angiography for cardiovascular events in patient presenting with chest pain. J Am Coll Cardiol 65: A1125.

29. Nørgaard KS, Isaksen C, Buhl JS, Kirk Johansen J, Nielsen AH, et al. (2015) Single-centre cohort study of gender influence in coronary CT angiography in patients with a low to intermediate pretest probability of coronary heart disease. Open Heart 2: e000233. 